\title{
Modelo de Intervención Epidemiológica y Psicológica para el Abordaje de los cuidados y precauciones poblacionales frente al Coronavirus (COVID-19)
}

Parra-Bolaños, Nicolás ${ }^{1(*)}$; Marulanda-Londoño, Leonardo Fabio²; Benjumea-Garcés, Juan Sebastián²

${ }^{1}$ Asociación Educar para el Desarrollo Humano, Laboratorio de Neurociencias y Educación, Buenos Aires, Argentina

²Institución Universitaria Digital de Antioquia, Vicerrectoría Académica, Medellín, Colombia

\section{RESUMEN}

El presente estudio de revisión documental, representa un esfuerzo por conseguir estructurar un modelo desde la psicología conductual y la epidemiología, adaptando el modelo de Luo, Gao \& Cassels (2018) a las realidades sociales de países en vías de desarrollo. Se ejecutaron dos softwares que son The Critical Appraisals Skills Programme - CASP y el Strengthening the Reporting of Observational Studies in Epidemiology - STROBE, con el objeto de configurar un diagrama de tipo PRISMA mediante cinco ecuaciones distintas para el ejercicio de bibliometría. Se revisaron 710.911 documentos de los Institutos Nacionales de la Salud de los Estados Unidos y se filtraron en un consolidado final de 26 artículos científicos mediante estrictos criterios de inclusión y exclusión. El prototipo de modelo psicológico y epidemiológico logró estructurarse en siete distintitas fases, demostrando ser consistente con los modelos existentes y podría servir como punto de partida para el diseño de proyectos regionales de contención de la COVID-19 en áreas de escasos recursos financieros y económicos destinados a combatir la pandemia.

Palabras clave: epidemiología; modelos Matemáticos; COVID-19; pandemia; bibliometría.

\section{Epidemiological and Psychological Intervention Model for Addressing Population Cares and Precautions against Coronavirus (COVID-19)}

\section{ABSTRACT}

The present review paper study represents an effort to structure a model from behavioral psychology and epidemiology, adapting the model of Luo, Gao \& Cassels (2018) to the social realities of developing countries. Two softwares were executed, which are The Critical Appraisals Skills Program - CASP and Strengthening the Reporting of Observational Studies in Epidemiology - STROBE, in order to configure a PRISMA-type diagram using five different equations for the bibliometrics exercise. 710,911 documents from the United States National Institutes of Health were reviewed and filtered in a final consolidation of 26 scientific articles using strict inclusion and exclusion criteria. The prototype of the psychological and epidemiological model was structured in seven distinct phases, proving to be consistent with the existing models and could serve as a starting point for the design of regional containment projects for COVID-19 in areas of limited financial and economic resources destined to fight the pandemic.

Keywords: epidemiology; mathematical Models; COVID-19; pandemic; bibliometry. 


\section{INTRODUCCIÓN}

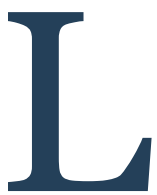

a epidemia clasificada como COVID-19, es un tipo de coronavirus que ha pasado de ser una enfermedad infecciosa solo con características epidémicas a una pandemia, llegando a afectar a casi la totalidad de países del mundo. Esta pandemia comenzó en la Ciudad de Wuhan en la República Popular China, extendiéndose por el resto de dicho país desde diciembre de 2019 y por más de cuatro meses en el resto del mundo, evidenciando alarmantes cifras de contagiados y a su vez, por fallecidos, haciendo que, durante el periodo del primer semestre de 2020, haya alcanzado a más de 180 naciones, presentando una alarmante cifra de más de 2.000.000 de contagiados, entre personas con cuadros clínicos sintomáticos y asintomáticos. Esta pandemia se expande en su primera ola por los cinco Continentes, no habiendo todavía marcadores predictivos para la segunda ola. La COVID-19 afecta con mayor impacto a sectores poblaciones ubicados entre los 60 y 80 años de edad que tengan alguna patología crónica o respiratoria, no queriendo decir con ello que, la COVID-19 no esté siendo letal en sectores poblacionales con otros rangos de edad o sin antecedentes médicos de enfermedad, pero ciertamente, en poblaciones infantiles, no ha evidenciado rasgos de mortandad elevados (Cucinotta \& Vanelli, 2020; Porcheddu, Serra, Kelvin, Kelvin \& Rubino, 2020; Velavan \& Meyer, 2020).

La tasa de crecimiento de la COVID-19, está demostrando ser exponencial en todos los modelos matemáticos que se basan en datos de conteo de pacientes, pero que no están logrando incorporar hallazgos provenientes de ciencias básicas en lo relativo a las peculiaridades fisiológicas de dicho virus, presentando, por ende, fallas en el máximo asintótico de las curvas que evalúan. Como consecuencia de la pandemia, se está evidenciando que, muchos sistemas de salud están al borde del colapso, pues carecen de medidas de control que cuiden, no solo al paciente infectado con COVID-19, sino también a todo el personal médico, lo que está representando un gasto financiero para todas las naciones, mucho más allá de sus rubros anuales destinados a salud. La falta de medidas económicas tempranas pasa subsanar situaciones como las pandemias, demuestra sus primeros efectos negativos en las naciones en vías de desarrollo, debido a que estas, son las que menos recursos poseen para poder mitigar los daños colaterales producidos por pandemias como la COVID-19. En el caso de las naciones más desarrolladas, estas se encuentran distribuyendo de forma ordenada, sistemática y progresiva, los recursos de sus arcas destinados a situaciones críticas, lo que comienza por la reorganización del sistema hospitalario, los mecanismos de diagnóstico para la COVID-19, los procedimientos de contención sociológica para sus respectivas poblaciones y por supuesto, la financiación y apoyo desmedidos a sus grupos de investigación en disciplinas científicas capaces de adelantar los diseños experimentales necesarios como para apresurar la puesta en marcha de fármacos, prototipos de vacunas y desarrollo de tecnologías capaces de desacelerar los daños provocados por la COVID-19 (Rao \& Vazquez, 2020; Vankadari \& Wilce, 2020; Zhuang, et al, 2020).

El objetivo del presente estudio de revisión documental, consistió en la elaboración de una pesquisa por las cuarenta bases de datos que conforman la gran Base de Datos de Ciencias Médicas de los Institutos Nacionales de la Salud o NIH por sus siglas en inglés, como punto de partida para rediseñar un modelo epidemiológico y psicológico que se pueda adaptar a contextos propios de naciones en vías de desarrollo que estén afectadas por la COVID-19. Cabe recalcar que, se ha optado por extraer la información de los NIH, puesto que estos manejan todos sus documentos científicos en PubMed, que básicamente es la matriz de información científica en ciencias de la salud, más grande del planeta. La revisión documental tuvo lugar al analizar las principales publicaciones sobre la COVID-19, mediante el empleo de dos programas bibliométricos especializados en dicha labor (Horesh \& Brown, 2020).

La enfermedad infecciosa conocida como COVID-19, comenzó siendo un brote epidémico menor, aparentemente, teniendo origen en algunas especies de murciélagos o pangolines, sin embargo, faltan más estudios que puedan verificar esta información con veracidad, pues hasta la fecha, no se tiene certeza de ello. Otro de los problemas graves que ha traído la COVID-19, es que, al ser parte de la familia de coronavirus, se la tomó como una simple gripa estacional, por lo que ahora, que ya se han venido identificando nuevas proteínas empaquetadas dentro de cada uno de estos mecanismos parasitarios de la COVID-19, es que se está comprendiendo que, es un virus altamente letal, ubicándolo con total seguridad, como la pandemia más destructiva de todo el siglo XXI (Shanmugaraj, Siriwattananon, Wangkanont \& Phoolcharoen, 2020).

Debido al enorme grado de complejidad que representa la COVID-19 para todas las ciencias básicas, 
es que esta clase de investigaciones se hacen cada vez más necesarias, dado que, la formación académica y/o científica de la población en general, evidencia escasas o nulas destrezas para interpretar los datos que a diario se publican en medios de comunicación, generando un halo de tensión, estrés, preocupación y ansiedad entre la mayor parte de personas que, la única forma que tienen de entender las dinámicas de la COVID-19, es por canales de televisión y/o por redes sociales, en donde se entremezclan los datos científicos con las percepciones subjetivas de algunos sectores poblaciones con capacidad de divulgación masiva de la información. Esto quiere decir que, se requiere del mayor grado posible de cualificación dentro de la población mundial, para hacerle frente a la COVID-19, no solo desde los laboratorios, sino también, desde las tareas que las ciencias sociales y humanas puedan adelantar para cuidar la salud mental de la población en tiempos de cuarentena y/o confinamientos prolongados y esa pretensión es uno de los objetivos esenciales de este estudio (Song \& Karako, 2020).

\section{MARCO TEÓRICO}

Los virus se definen desde la biología convencional como mecanismos autorreplicantes, que se caracterizan por ser agentes infecciosos incapaces de contar con metabolismos propios, -que es lo que hace que la biología no les pueda conferir una taxonomía como especies animales-, lo que no quiere decir que, por ello, sean mucho menos complejos que los organismos animales como los mamíferos. Por tanto, los diferentes tipos de virus se clasifican como agentes parasitarios acelulares, con la destreza de ingresar a células de origen vegetal o animal, para aprovechar la capacidad metabólica de dichas células, pudiendo destruirlas desde dentro y logrando replicarse a su vez, en cientos de miles de agentes dentro de una única célula, por lo que, cualquier virus, posee amplias posibilidades de infectar, no solo a su huésped, sino a otros organismos propios de la especie infectada e incluso, terminar afectando ecosistemas completos (Gao, Tian \& Yang, 2020).

Diversas disciplinas científicas están trabajando para entender la pandemia desde diciembre de 2019, fecha en que fue descubierta la COVID-19, permitiendo que, desde aquel entonces hasta el primer semestre de 2020, se estén sumando esfuerzos de miles y miles de investigadores de todo el mundo, para concretar herramientas de ayuda a la población, bien sea desde las ciencias naturales, ciencias exactas y ciencias sociales, con el objeto de aumentar el cerco epidemiológico contra la COVID-19. En esta línea de esfuerzos científicos, también se observan múltiples estudios realizados desde el área de la psicología, tales como los llevados a cabo por Wang, Zhang, Zhao, Zhang \& Jiang (2020), logrando detectarse, al menos media docena de publicaciones en las que, desde una psicología basada en la evidencia, ya es posible contar con herramientas de diagnóstico temprano de cuadros clínicos de salud mental por causa de prolongados periodos de tiempo en que las diferentes poblaciones han tenido que guardar estrictas cuarentenas y también se han identificado tratamientos psicológicos en tiempos de pandemia (Asmundson \& Taylor, 2020; Chen, et al, 2020; Hoffmann, et al, 2020; Liu, et al, 2020; Wujtewicz, et al, 2020; Yang, et al, 2020).

Abordando las aportaciones que la psicología está consiguiendo hacer para beneficiar a la población mundial con estrategias, herramientas y técnicas que están en condiciones de contribuir a mejorar la salud mental de la población, es más que evidente que, los encierros prolongados generan situaciones adversas en el ser humano. Debido a esto, es que se ha optado por rescatar los avances de autores como Duan \& Zhu (2020), en materia de intervenciones psicológicas a poblaciones afectadas por la COVID-19, además de los modelos propios de la Terapia de Aceptación y Compromiso - ACT (Hofmann \& Hayes, 2019), añadiendo los modelos de interacción geo-social para temporadas de brotes y epidemias, expuestos por Luo, Gao \& Cassels (2018).

Los presupuestos conceptuales de la Teoría de Marcos Relaciones o RFT por sus siglas en inglés, ofrecen una ventaja para que expertos en epidemiología, psicología social y sociología, puedan ejecutar modelos en los que se posibilite la generación de estrategias de contención psicológica, tanto para atención temprana del estrés y la ansiedad en la población, así como en casos de depresión y problemas de violencia intrafamiliar, dado que, las prolongadas cuarentenas, por causa de la COVID-19, tienden a producir secuelas psicológicas como las enunciadas. La RFT, aporta elementos sólidos sobre cómo los seres humanos tienen una vida prosocial y sobre cómo, según los intereses y particularidades de cada individuo, tendemos naturalmente a formar parte de aquellos grupos con los cuales tenemos mayor familiaridad, sentido de pertenencia y afiliación (Atkins, Wilson \& Hayes, 2019). 
Una de las principales fortalezas que ofrece la RFT es que da a luz a la Terapia de Aceptación y Compromiso, más conocida por sus siglas en inglés como $A C T$, siendo ambas -RFT y ACT-, términos propios de las corrientes conductuales de tercera generación o tercera ola. ACT consiste en un tipo de terapia que envuelve las más modernas técnicas conductuales y cognitivas para tratamientos propios de psicología clínica, siendo especialmente fuerte en consolidarse como una psicología basada en la evidencia, dedicando sus esfuerzos a la creación de métodos clínicos para el tratamiento de la ansiedad, las fobias, el estrés, las ideaciones suicidas, las rumias, trastornos del estado del ánimo, problemas emocionales y afectivos, entre muchos otros, pero siempre, respaldando sus progresos en diseños experimentales probados, replicables y medibles (Gil-Luciano, Calderón-Hurtado, Tovar, Sebastián \& Ruiz, 2019).

\section{METODOLOGÍA}

Se ha elaborado un procedimiento de bibliometría, empleando un rastreo a todas las bases de datos de los Institutos Nacionales de la Salud de los Estados Unidos de Norteamérica, puesto que conforman un conjunto de 40 bases clasificadas en los más altos cuartiles, confiriéndoles un alto grado de rigurosidad científica, confiabilidad, además de validez interna y externa. Esas 40 bases de datos, integran el motor de búsqueda conocido como PubMed, que es un banco de información para ciencias de la salud que cubre todas las disciplinas científicas basadas en la evidencia y en metodologías experimentales, clínicas, convencionales y también de punta, haciendo de PubMed, uno de los motores de búsqueda con la mayor red mundial de datos médicos y epidemiológicos en el planeta en tópicos de salud.

Para la recogida de datos se han empleado los softwares conocidos como: The Critical Appraisals Skills Programme - CASP y el Strengthening the Reporting of Observational Studies in Epidemiology - STROBE, ya que son dos programas que permiten recoger todas las publicaciones científicas en grandes matrices de datos, condensarlas y mediante algorítmica, hacer una identificación, preselección, selección e inclusión de los artículos científicos que cumplen con los criterios de inclusión y exclusión del estudio de revisión documental ejecutado, además de constituir un esqueleto de datos coherentes con el título de este estudio. Las publicaciones científicas retomadas del motor de búsqueda PubMed, se registran mediante un Diagrama de tipo PRISMA (VuNgoc, et al, 2018), debido a que es una herramienta ampliamente usada por las ciencias de la salud para estudios de revisión o meta-análisis y presenta la información de forma jerárquica a manera de embudo hasta consolidar un total definido paramétricamente para estudios sistemáticos de literatura científica.

Los criterios de inclusión y exclusión, son parte esencial de todos los estudios soportados en la evidencia, por lo cual, este tipo de manuscrito no es la excepción a esa regla, concibiendo como criterios de inclusión, las siguientes aristas: a) Solo tomar estudios clínicos; b) Solo tomar estudios con muestras representativas; c) Solo tomar estudios con diseños experimentales claramente definidos en sus pasos; d) Solo tomar estudios con metodologías enfocadas en ciencias de la salud y e) Solo tomar estudios con alta fiabilidad estadística y con aplicaciones concretas en poblaciones humanas. En cuanto a los criterios de exclusión, estos fueron los siguientes: I) Se rechazaron estudios con muestras demasiado pequeñas; II) Se rechazaron artículos de reflexión, artículos de revisión, cartas el Editor y trabajos de grado; III) Se rechazaron estudios sin Abstract y estudios con deficiencias metodológicas; IV) Se rechazaron estudios con escasa fiabilidad estadística y V) Se rechazaron todos los estudios con temáticas no asociadas.

Los softwares CASP y STROBE hicieron un rastreo detallado de publicaciones disponibles en las bases de datos de los NIH, la cual es PubMed, bajo cinco ecuaciones que fueron: "Virus, Epidemic, Pandemic, Coronavirus, COVID-19". Estas se convirtieron de manera individual en ecuaciones de búsqueda para los años comprendidos entre 2016, 2017, 2018, 2019 hasta el 13 de abril de 2020. La correlación entre los términos seleccionados se hizo con el respaldo de los filtros de localización proporcionados por las herramientas del motor de búsqueda de PubMed, arrojando datos concretos como los que se pueden observar en la Tabla 1. 
Tabla 1. Condensado por matrices de la bibliometría hecha con CASP y STROBE

\begin{tabular}{c|c|c}
\hline Ecuación & Base de Datos & Total \\
\hline Virus & PubMed & 172,184 \\
\hline Epidemic & PubMed & 527,045 \\
\hline Pandemic & PubMed & 5,916 \\
\hline Coronavirus & PubMed & 4,355 \\
\hline COVID-19 & PubMed & 1,401 \\
\hline
\end{tabular}

Nota. Fuente: Elaboración propia (2020)

En la Tabla 1 se puede denotar que, las cinco ecuaciones están relacionadas entre sí en función de conceptos claves para la epidemiología, lo que es un rasgo decisivo para la propuesta de modelo que se hace en este manuscrito, pues el diseño de modelos, debe conllevar un rastreo bibliométrico completo y respaldado en criterios de rigor científico. En la columna de la izquierda aparecen las cinco ecuaciones empleadas, en tanto que, en la columna de centro se observa que, las publicaciones rastreadas se corresponden con las 40 bases de datos de PubMed y en la columna de la derecha se observa el número total del rastreo inicial hecho en dichas bases de datos.

En la Figura 1 se emplea un diagrama de tipo PRISMA, pues es una metodología ampliamente usada en la actualidad por autores como Guiu-Tula, Cabanas-Valdés, Sitjà-Rabert, Urrútia \& Gómara-Toldrà (2017), pues considera un amplio espectro de temáticas que se pueden alinear en una única columna vertebral de datos, como lo han hecho Zhang, Huang \& Du (2017), y permite la obtención de matrices con datos que responden a planteamientos de investigaciones documentales que requieran decantar la información de forma precisa, sólida y coherente, tal y como lo evidencian autores como $\mathrm{Vu}-\mathrm{Ngoc}$, et al (2018).
Figura 1. Diagrama de Tipo PRISMA para el ejercicio bibliométrico realizado con CASP y STROBE.

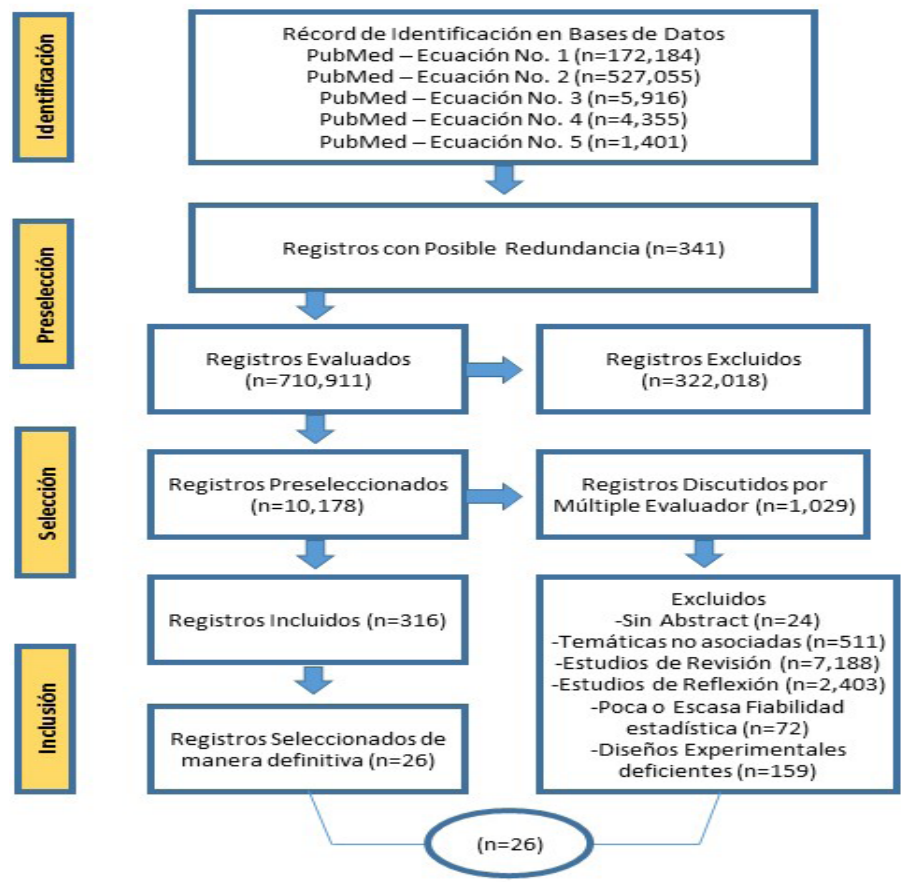

Nota. Fuente: Elaboración propia (2020).

En la Figura 1 se denotan en el extremo de la izquierda, cuatro recuadros, que son los que enmarcan los respectivos procedimientos que se realizaron con los softwares CASP y STROBE y que se delimitan en nueve recuadros que aparecen en la parte central y derecha del diagrama de tipo PRISMA con bordes azules, en tanto que al final de dichos recuadros, aparece la suma final consolidada en un ovalo, el cual, presenta la cifra total de artículos científicos empleados para este estudio bibliométrico. Cabe recalcar que, la totalidad de referencias bibliográficas recogidas para la construcción de todos y cada uno de los apartados del presente manuscrito, son las que se corresponden con lo representando en dicho ovalo a nivel numérico.

\section{RESULTADOS Y/O DISCUSIÓN}

El modelo presentado en este manuscrito, es una adaptación del modelo de Luo, Gao \& Cassels (2018), debido a que presenta múltiples ventajas para el manejo de las redes, los escenarios de control, la estandarización de datos para cada subregión y los análisis sectoriales como eje primordial de comprensión de las dinámicas epidemiológicas de la COVID-19 en cada ciudad del mundo, pues debido a las características genéticas y dietarias de cada grupo humano que habite las zonas afectadas por la COVID-19, 
se hace necesario un modelo capaz de procesar la información en paralelo con otras disciplinas científicas además de la epidemiología, en donde los programas de tiempo sean llevados a cabo hora tras hora, para permitir el mapeo y la geolocalización con principios sociales sobre las modificaciones y permutaciones que puede presentar la COVID-19 al correlacionarse con otros cuadros clínicos y con otros ambientes que puedan favorecer o acelerar la curva de crecimiento de dicho virus.

La Figura 2, presenta la adaptación del modelo de Luo, Gao \& Cassels (2018), la cual se divide en siete fases, siendo la primera de ellas, la medida de red (intermediación), que tiene como propósito, hacer una evaluación preliminar de los datos sociodemográficos de las poblaciones a evaluar. La segunda fase consiste en análisis sectoriales, haciendo cruce de la información que manejan las diferentes secretarías locales, mediante actualizaciones de datos que sean de 30 a 40 horas de revisión y cotejado de dichos datos.

La tercera fase se constituye por las redes sociales basadas en la movilidad de los individuos, lo que quiere decir que, se deben hacer una completa geolocalización de la movilidad ciudadana, zona por zona, generando modelos computacionales que permitan hacer el mapeo, preferiblemente con observaciones de campo, sistemas de flotas de drones y sensórica para garantizar la fidelidad de los mapeos. La cuarta fase son las redes basadas en ubicación, las que estarían permitiendo el diseño de escenarios de control, al tomar como punto de partida, las dinámicas de movimiento de la ciudadanía dentro de áreas urbanas y rurales. La quinta fase, se constituye en el diseño de escenarios basados en patrones de interacción geo-social, lo que debe ofrecer una cantidad masiva de datos relativos a los lugares y horas de mayor movilización ciudadana, sirviendo como referente para disminuir al máximo la movilidad de agentes como la COVID-19.

La sexta fase es el modelo epidemiológico basado en agentes infecciosos, que permitiría a los expertos en movilidad ciudadana y servicios públicos, cruzar la información entre las características arquitectónicas de las ciudades y los momentos de desplazamiento de la ciudadanía de un punto a otro, para así, contar con datos geolocalizados sobre las dinámicas de desplazamiento ciudadano durante el tiempo de incubación de la COVID-19 y en momentos posteriores. La séptima fase consiste en aumentar las horas semanales de escenarios de control, pasando de 10 horas a 60 horas, para lo cual se requiere de todos los grandes ordenadores de los que disponga cada ciudad, en aras de tener una analítica de datos y un procesamiento de la información mediante Big-Data, que arroje información precisa sobre el comportamiento, el desarrollo, la evolución y las medidas de control propias de cada entorno o región, para frenar la curva de crecimiento de la COVID-19.

Las siete fases descritas, hacen alusión al modelo que se ha adaptado de Luo, Gao \& Cassels (2018), lo que incluye la innovación social de considerar las aportaciones de RFT y ACT, como productos devenidos de la psicología contextualista, que pueden ponerse fácilmente en función del modelo mencionado, pues son un área de la psicología, eminentemente respaldadas por las evidencias clínicas y experimentales, confiriéndoles así, las condiciones necesarias como para amalgamarse con el modelo diseñado en este estudio.

Figura 2. Modelo de Intervención Psicológica y Epidemiológica para el Abordaje de los cuidados y precauciones poblacionales frente al Coronavirus (COVID-19). Adaptado de: Luo, Gao \& Cassels (2018).

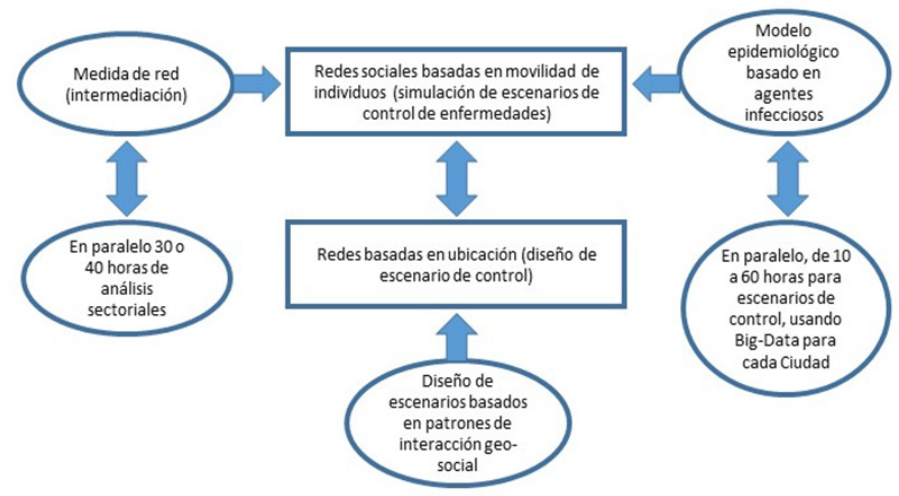

Nota. Fuente: Elaboración propia (2020).

El modelo opuesto al que se propone en este modelo es el diseñado por Lin et al (2020), dado que es una propuesta sumamente lineal, dando lugar a una única línea de tiempo de evolución y desarrollo de la COVID-19, lo que, desde la perspectiva de este manuscrito, resulta ser un error por omisión, pues si algo ha demostrado la puesta en escena de la COVID-19, es la necesidad de aunar esfuerzos entre diferentes disciplinas científicas en aras de alcanzar modelos epidemiológicos mucho más centrados en la posibilidad de mutar que presenta la COVID-19 en los multivariados entornos en donde habita el ser humano.

La adaptación que se ha hecho en el modelo presentado en este manuscrito, tiene como meta, que naciones en vías de desarrollo, puedan usar el sistema de flujo de información que aparece en el diagrama, con el objeto de llevarlo a la práctica con las herra- 
mientas preexistentes a la pandemia de la COVID-19, por lo que, las tecnologías ingenieriles con las que cuentan las universidades, los laboratorios y los softwares de grandes sistemas públicos y bancarios, estarían en condiciones de adaptar sus plataformas virtuales para el procesamiento de la información mediante Big-Data, haciendo que, las masas de datos relativas a potenciales poblaciones a contagiarse de la COVID-19, puedan cotejarse con el número real de infectados, el número de fallecidos, las medidas de confinamiento y aislamiento tomadas por el Estado, además de las prácticas de salubridad, higiene y bioseguridad, para entregar información que se pueda sistematizar bajo el modelo aquí sustentado.

Se señala de manera clara y concreta que, el modelo expuesto en este manuscrito, debe incluir en sus siete fases, un procedimiento concomitante, devenido de la teoría Prosocial de Atkins, Wilson \& Hayes (2019), debido a que, es un presupuesto que guarda compatibilidad con el modelo presentado, razón por la cual, para cada una de las fases, se recomiendan los siguientes pasos: 1) Incentivar el fortalecimiento social dentro de pequeños grupos de no más de una treintena de personas en todas cada una de las áreas geográficas en las que se aplique el modelo; 2 ) Generar propuestas educativas y/o formativas para que las diferentes poblaciones puedan conformar pequeños grupos de instrucción y regulación en lo relativo a información confiable sobre la COVID-19; 3) Una vez conformados esos pequeños grupos, promover en ellos, la toma de decisiones pensando en el bienestar colectivo; 4) Hacer monitoreos semanales sobre la salud física y sobretodo mental de cada miembro del grupo mediante aplicativos y teletrabajo ejercido por profesionales de la psicología y demás ciencias del comportamiento cualificados para atender casos específicos; 5) Establecer un sistema de reforzamiento y sanciones dentro de cada grupo, llevando control del mismo, mediante formatos cortos y prácticos que puedan entregarse y explicarse a dichos grupos, permitiendo el seguimiento y evaluación de la salud mental de cada pequeño grupo; 6) Diseñar mecanismos de resolución de conflictos y de apoyo mutuo dentro de cada grupo, para así fortalecer la naturalización de herramientas conductuales al interior de cada equipo de trabajo y 7) Evaluar los casos más exitosos de manejo del estrés, ansiedad y conflictos en los grupos, para generar intercomunicación entre grupos altamente exitosos y grupos con bajo margen de éxito, de modo que se puedan intercambiar información, para contribuir no solo al manejo social de la COVID-19, sino también, para evitar brotes de problemas de salud mental con características epidémicas o pandémicas.

\section{CONCLUSIONES}

Los modelos matemáticos que se están empleando actualmente para predecir las curvas de crecimiento de la COVID-19, poseen solo rasgos paramétricos basados en la epidemiología, lo que debería entrar a corregirse pues, en tiempos de pandemia, las diferentes disciplinas científicas deben unirse por la consecución de un mismo objetivo. Esto quiere decir que, si se esperan diseñar modelos matemáticos eficaces para la COVID-19, debe hacerse un análisis enfocado no en la forma en que se mueve la pandemia en otros países, sino en las mutaciones que posee en cada nación, en su evolución, su desarrollo y sobretodo: en las características genéticas y contextuales de cada población. Ello con el objeto de disminuir al máximo posible el margen de error, posibilitando de ese modo, la gestación de modelos matemáticos respaldados por disciplinas como la inmunología, la virología, la biología molecular, la microbiología, la inmunoterapia, la ingeniería genética, la proteómica, la química y claro está, la epidemiologia.

El uso de tecnologías emergentes debe concatenarse con las tecnologías preexistentes en los sistemas hospitalarios, pues no hacerlo, representaría un gasto descomunal del PIB de cada nación, lo que afectará a economías de países en vías de desarrollo de forma inexorable, dada la falta de desarrollo en nuevas patentes en el área de estudios de los coronavirus, en regiones como África y Latinoamérica, en donde no existe un rubro destinado en varios miles de millones de USD anuales para la investigación de agentes patógenos y para la producción masiva de biotecnologías. Esta falencia claramente visible en todas las naciones de las dos regiones antes mencionadas, hace que sean dependientes de los desarrollos tecnológicos de las naciones más desarrolladas, lo que es una dependencia sumamente perjudicial en ciencias de la salud.

Por último, se recomienda que, se emplee el modelo sugerido, específicamente en naciones con pocos recursos tecnológicos, científicos y financieros, dado que, es un modelo que se ha diseñado pensando en naciones en vías de desarrollo, por lo que, si se parte del hecho de que el confinamiento es una condición necesaria para el éxito del modelo, también lo es la coordinación que los sectores públicos y privados puedan tener para implementar el modelo, generando con ello, un aumento significativo en el cuidado 
de la salud mental de las familias y de las personas en situación de confinamiento prolongado, pues con el respaldo del componente psicológico, el componente epidemiológico podrá ser mucho más efectivo en sus extensiones y aplicaciones. 


\section{REFERENCIAS}

Asmundson, G., \& Taylor, S. (2020). How health anxiety influences responses to viral outbreaks like COVID-19: What all decision-makers, health authorities, and health care professionals need to know. Journal of Anxiety Disorders, 71:102211. https://doi.org/10.1016/j.janxdis.2020.102211

Atkins, W. B., Wilson, D. S., \& Hayes, S. C. (2019). Prosocial: Using evolutionary science to build productive, equitable, and collaborative groups. Context Press. New Harbinger Publications, Inc. Oakland, CA.

Chen, Q., et al. (2020). Mental health care for medical staff in China during the COVID-19 outbreak. Lancet Psychiatry, 7(4), e15-e16. https://doi. org/10.1016/S2215-0366(20)30078-X

Cucinotta, D., \& Vanelli, M. (2020). WHO Declares COVID-19 a Pandemic. Acta Biomedica, 91(1), 157160. https://doi.org/10.23750/abm.v91i1.9397

Duan, L., \& Zhu, G. (2020). Psychological interventions for people affected by the COVID-19 epidemic. The Lancet Psychiatry, 7(4), 300-302. https://doi. org/10.1016/s2215-0366(20)30073-0

Gao, J., Tian, Z., \& Yang, X. (2020). Breakthrough: Chloroquine phosphate has shown apparent efficacy in treatment of COVID-19 associated pneumonia in clinical studies. Bioscience Trends, 14(1), 72-73. https://doi.org/10.5582/bst.2020.01047

Gil-Luciano, B., Calderón-Hurtado, T., Tovar, D., Sebastián, B., \& Ruiz, F. J. (2019). How are triggers for repetitive negative thinking organized? A relational frame analysis. Psicothema, 31(1), 53-59. https:// doi.org/10.7334/psicothema2018.133

Guiu-Tula, F., Cabanas-Valdés, R., Sitjà-Rabert, M., Urrútia, G., \& Gómara-Toldrà, N. (2017). The Efficacy of the proprioceptive neuromuscular facilitation (PNF) approach in stroke rehabilitation to improve basic activities of daily living and quality of life: a systematic review and meta-analysis protocol. $B M J$ Open, 7(12), e016739. https://doi.org/10.1136/ bmjopen-2017-016739

Hoffmann M., et al. (2020). SARS-CoV-2 Cell Entry Depends on ACE2 and TMPRSS2 and Is Blocked by a Clinically Proven Protease Inhibitor. Cell, S00928674(20), 30229-4. https://doi.org/10.1016/j. cell.2020.02.052

Hofmann, S. G., \& Hayes, S. C. (2019). The Future of Intervention Science: Process-Based Therapy. Clinical Psychological Science, 7(1), 37-50. https://doi. org/10.1177/2167702618772296

Horesh, D., \& Brown, A. (2020). Traumatic stress in the age of COVID-19: A call to close critical gaps and adapt
Lin, Q., et al. (2020). A conceptual model for the coronavirus disease 2019 (COVID-19) outbreak in Wuhan, China with individual reaction and governmental action. International Journal of Infectious Diseases, 93, 211-216. https://doi.org/10.1016/j. ijid.2020.02.058

Liu, S., et al. (2020). Online mental health services in China during the COVID-19 outbreak. Lancet Psychiatry, 7(4), e17-e18. https://doi.org/10.1016/S22150366(20)30077-8

Luo, W., Gao, P., \& Cassels, S. (2018). A large-scale location-based social network to understanding the impact of human geo-social interaction patterns on vaccination strategies in an urbanized area. Computers, Environment and Urban Systems, 72, 78-87. https://doi.org/10.1016/j.compenvurbsys.2018.06.008

Porcheddu, R., Serra, C., Kelvin, D., Kelvin, N., \& Rubino, S. (2020). Similarity in Case Fatality Rates (CFR) of COVID-19/SARS-COV-2 in Italy and China. Journal of Infection in Developing Countries, 14(2), 125128. https://doi.org/10.3855/jidc.12600

Rao, A., \& Vazquez, J. (2020). Identification of COVID-19 Can be Quicker through Artificial Intelligence framework using a Mobile Phone-Based Survey in the Populations when Cities/Towns Are Under Quarantine. Infection Control and Hospital Epidemiology, 3, 1-18. https://doi.org/10.1017/ ice.2020.61

Shanmugaraj, B., Siriwattananon, K., Wangkanont, K., \& Phoolcharoen, W. (2020). Perspectives on monoclonal antibody therapy as potential therapeutic intervention for Coronavirus disease-19 (COVID-19). Asian Pacific Journal of Allergy and Immunology, 38(1), 10-18. https://doi. org/10.12932/AP-200220-0773

Song, P., \& Karako, T. (2020). COVID-19: Real-time dissemination of scientific information to fight a public health emergency of international concern. Bioscience Trends, 14(1), 1-2. https://doi.org/10.5582/ bst.2020.01056

Vankadari, N., \& Wilce, J. (2020). Emerging WuHan (COVID-19) coronavirus: glycan shield and structure prediction of spike glycoprotein and its interaction with human CD26. Emerging Microbes \& Infections, 9(1), 601-604. https://doi.org/10.1080 /22221751.2020.1739565

Velavan, T., \& Meyer, C. (2020). The COVID-19 epidemic. Tropical Medicine and International Health, 25(3), 278-280. https://doi.org/10.1111/tmi.13383

Vu-Ngoc, H., et al. (2020). Quality of flow diagram in systematic review and/or meta-analysis. PLoS One, 
13(6), e0195955. https://doi.org/10.1371/jour-

nal.pone.0195955

Wang, G., Zhang, Y., Zhao, J., Zhang, J., \& Jiang, F. (2020). Mitigate the effects of home confinement on children during the COVID-19 outbreak. Lancet, 395(10228), 945-947. https://doi.org/10.1016/ S0140-6736(20)30547-X

Wujtewicz, M., et al. (2020). COVID-19 - what should anaethesiologists and intensivists know about it? Anaesthesiology Intensive Therapy, 52(1), 34-41. https://doi.org/10.5114/ait.2020.93756

Yang, Y., et al. (2020). Mental health services for older adults in China during the COVID-19 outbreak. Lancet Psychiatry, 7(4), e19. https://doi. org/10.1016/S2215-0366(20)30079-1

Zhang, Y., Huang, J., \& Du, L. (2017). The top-cited systematic reviews/meta-analyses in tuberculosis research: A PRISMA-compliant systematic literature review and bibliometric analysis. Medicine, 96(6), e4822. https://doi.org/10.1097/ MD. 0000000000004822

Zhuang, Z., et al. (2020). Preliminary estimation of the novel coronavirus disease (COVID-19) cases in Iran: A modelling analysis based on overseas cases and air travel data. International Journal of Infectious Diseases, S1201-9712(20), 30138-7. https://doi. org/10.1016/j.ijid.2020.03.019 